\title{
Morphological Characteristics of Compact High-Velocity Clouds Revealed by High-Resolution WSRT Imaging
}

\author{
W. B. Burton \\ Sterrewacht Leiden, P.O. Box 9513, 2300 RA Leiden, The Netherlands \\ R. Braun \\ Netherlands Foundation for Research in Astronomy, P.O. Box 2, 7990 \\ AA Dwingeloo, The Netherlands
}

\begin{abstract}
A class of compact, isolated high-velocity clouds which plausibly represents a homogeneous subsample of the HVC phenomenon in a single physical state was objectively identified by Braun and Burton (1999). Six examples of the CHVCs, unresolved in single-dish data, have been imaged with the Westerbork Synthesis Radio Telescope. The highresolution imaging reveals the morphology of these objects, including a core/halo distribution of fluxes, signatures of rotation indicating dark matter, and narrow linewidths constraining the kinetic temperature of several opaque cores. In these regards, as well as in their kinematic and spatial deployment on the sky, the CHVC objects are evidently a dynamically cold ensemble of dark-matter-dominated $\mathrm{H}$ I clouds accreting onto the Local Group in a continuing process of galactic evolution.
\end{abstract}

\section{Introduction}

The criteria applied by Braun and Burton (1999) to the Leiden/Dwingeloo H I Survey of Hartmann \& Burton (1997) and the HVC catalog of Wakker \& van Woerden (1991) led to a catalog of 65 confirmed examples of compact, isolated high-velocity clouds. The selection criteria excluded the Magellanic Stream and all other HVC complexes. The catalog is more likely to represent a single phenomenon than would a sample which included the major HVC complexes. The CHVC objects plausibly originated under common circumstances, have shared a common evolutionary history, are arguably in a single physical state, and have not (yet) been strongly influenced by the radiation field of the Milky Way or of M31, or by a gravitational encounter with one of these major systems. In this context, the extended HVC complexes would be the nearby objects currently undergoing accretion onto the Galaxy, while the more compact, isolated ones would be their distant counterparts in the Local Group environment. The positional and kinematic characteristics of the compact HVCs are similar in many regards to those of the Local Group galaxies. The sample is distributed quite uniformly over the sky, and defines a well-organized kinematic system. The kinematic signature of this system suggests an in-falling population associated with the Local Group gravitational potential. 

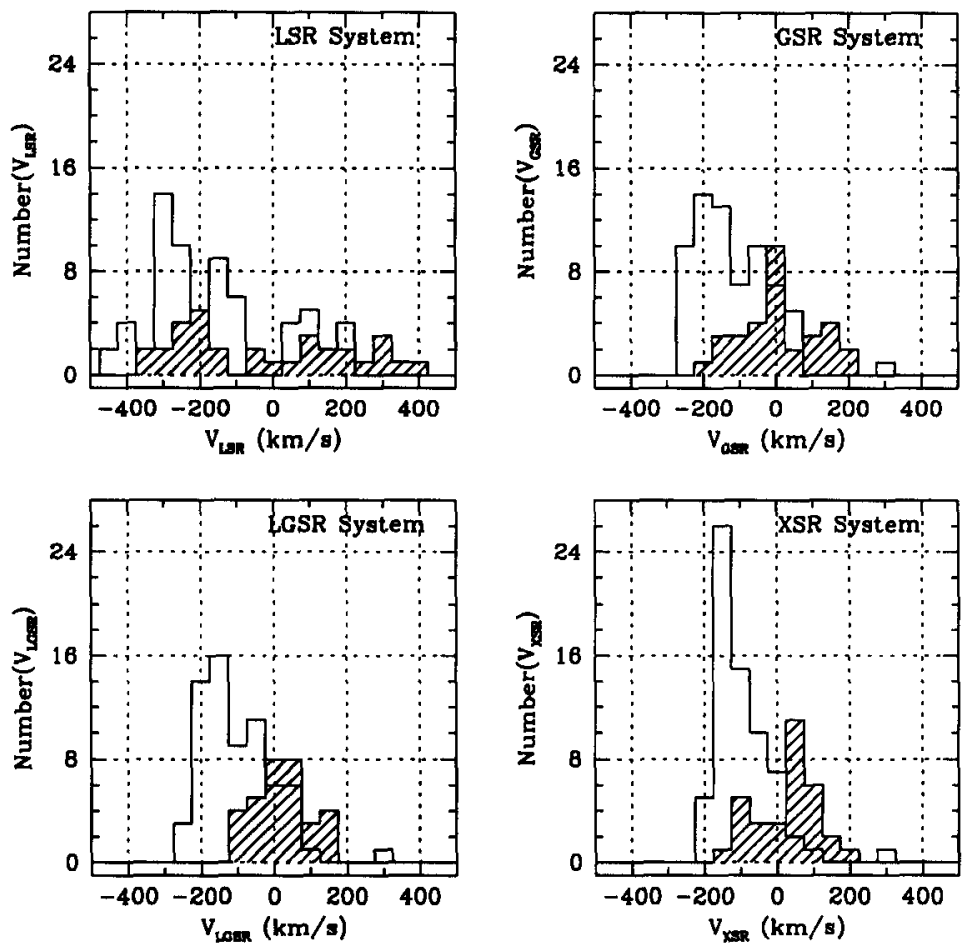

Figure 1. Histograms of distributions of the CHVC velocities, and of Local Group galaxy velocities, as measured in the indicated reference frames (Braun and Burton 1999). The solid lines represent the CHVC ensemble; the shaded areas, Local Group galaxies. The dispersion in the $v_{\mathrm{LGSR}}$ frame is significantly smaller than those in the $v_{\mathrm{LSR}}$ and $v_{\mathrm{GSR}}$ reference frames. The velocities labelled $v_{\mathrm{XSR}}$ refer to a frame which minimizes the dispersion of the CHVC velocity distribution; within the errors, this reference frame is consistent with the Local Group Standard of Rest. This minimization provides a quantitative demonstration of Local-Group deployment. Within this frame, the CHVC ensemble is dynamically quite cold, with a dispersion of only $69 \mathrm{~km} \mathrm{~s}^{-1}$, although strongly in-falling into the Local Group barycenter at a velocity of about $100 \mathrm{~km} \mathrm{~s}^{-1}$. 


\section{Minimized Velocity Dispersion of the CHVC Ensemble as an Indication of Local Group Deployment}

The possibility of the extragalactic nature of high-velocity clouds has been considered in various contexts by, among others, Oort (1966, 1970, 1981), Verschuur (1975), Eichler (1976), Einasto et al. (1976), Giovanelli (1981), Bajaja et al. (1987), Burton (1997), Wakker and van Woerden (1997), Braun and Burton (1999), and Blitz et al. (1999). Nevertheless, no direct distance determination is yet available for any of the individual CHVC objects. Distances are, of course, required to establish the values of important physical parameters: linear size varies as $D$, mass as $D^{2}$, and density as $D^{-1}$.

Several aspects of the topology of the class are difficult to account for if the CHVCs are viewed as a Milky Way population, in particular if they are viewed as consequences of a galactic fountain. The amplitude of the horizontal motions of these "bullets" is comparable to that of the vertical motions. The vertical motions are larger than expected for free fall onto the Milky Way from material returning in a fountain flow. The topology shows no preference for the terminal-velocity locus, where motions from violent events leading to a fountain would be expected to be most common. Unlike the situation if the major HVC complexes are considered, the CHVCs are scattered rather uniformly across the sky. The CHVCs show no tendency to accumulate in the lower halo of the Milky Way.

On the other hand, regarding both their spatial and kinematic distributions, the CHVCs show substantial similarities with the distributions of the galaxies comprising the Local Group. Since our CHVC sample has both a substantial size and a rather uniform distribution on the sky (see Fig. 1 of Braun and Burton 1999) it is appropriate to use the sample itself to define a best-fitting velocity reference system. Braun and Burton (1999) showed that the velocity dispersion of the ensemble is minimized in a reference frame consistent with the Local Group Standard of Rest. The solar apex which follows directly from a minimization of the velocity dispersion of the CHVC system, namely, $\left(l_{\odot}, b_{\odot}, v_{\odot}\right)=$ $\left(88^{\circ},-19^{\circ},+293 \mathrm{~km} \mathrm{~s}^{-1}\right)$, agrees within the errors with that which defines the Local Group Standard of Rest, $\left(l_{\odot}, b_{\odot}, v_{\odot}\right)=\left(93^{\circ},-4^{\circ},+316 \mathrm{~km} \mathrm{~s}^{-1}\right)$, found by Karachentsev \& Makarov (1996). The velocity dispersion of the CHVC system in this reference frame is only $\sigma_{\mathrm{XSR}}=69 \mathrm{~km} \mathrm{~s}^{-1}$, while there is a mean in-fall of $v_{\mathrm{LGSR}}=v_{\mathrm{XSR}}=-100 \mathrm{~km} \mathrm{~s}^{-1}$.

Figure 1 shows histograms of the velocities in these reference frames. The dispersion of the velocities decreases in a progression from the $v_{\mathrm{LSR}}$ reference frame, for which $\sigma_{\mathrm{LSR}}=175 \mathrm{~km} \mathrm{~s}^{-1}$, via the $v_{\mathrm{GSR}}\left(\sigma_{\mathrm{GSR}}=95 \mathrm{~km} \mathrm{~s}^{-1}\right)$ and the $v_{\mathrm{LGSR}} \sigma_{\mathrm{LGSR}}=88 \mathrm{~km} \mathrm{~s}^{-1}$ frames, to the minimum of $\sigma_{\mathrm{XSR}}=69 \mathrm{~km} \mathrm{~s}^{-1}$ for the $v_{\mathrm{XSR}}$ frame. This minimization provides a quantitative demonstration of LocalGroup deployment and, in addition, that the CHVC ensemble is dynamically quite cold.

\section{Imaging with the WSRT}

The CHVC objects catalogued by Braun and Burton (1999) are not spatially resolved in the single-dish data of the Leiden/Dwingeloo Survey. Insofar as the 
objects have not been resolved in angle, it has remained ambiguous if the singledish linewidth refers to the intrinsic characteristics of a single entity or to the collective behavior of blended features. High-resolution imaging is required to reveal such kinematic properties as intrinsic linewidths and opacity information, or indications of rotational support requiring a higher total mass than available in the $\mathrm{HI}$ alone, as well as the resolved structural morphology. If the CHVCs are in fact a population of primordial clouds scattered throughout the Local Group, then they might reveal some morphological characteristics which would not be consistent with the expectations of other suggested scenarios, in particular for objects ejected by a galactic fountain (e.g. Shapiro and Field 1976, Bregman 1980 ) or located within the Galactic halo (Moore et al. 1999). High-resolution imaging is also necessary to provide specific targets for deep optical observations. Such optical probes would help clarify the distinction between the CHVCs and (sub-)dwarf galaxies, and any indication of a stellar population would offer a distance indication.

Of the sample of 65 compact, isolated HVCs catalogued by Braun and Burton (1999), only two had been subject to interferometric imaging. Wakker and Schwarz (1991) used the Westerbork array to show that both CHVC114-10-430 and CHVC111-06-466 are characterized by a core/halo morphology, with only about $40 \%$ of the single-dish flux recovered on angular scales of tens of arcmin, and, furthermore, that the linewidths of the single-dish spectra of these two sources were resolved into components of some $5 \mathrm{~km} \mathrm{~s}^{-1}$ width or less. Both of the imaged systems display systematic velocity gradients along the major axis of an elliptical $\mathrm{H}$ I distribution, which Wakker and Schwarz judged to be suggestive of rotation in self-gravitating systems at Local Group distances.

Braun and Burton (2000) have used the Westerbork array to image an additional six objects of the CHVC class. Although only six CHVC sources were imaged in our program, the sources are distributed widely in galactic coordinates, span radial velocities of $-275<v_{\mathrm{LSR}}<+165 \mathrm{~km} \mathrm{~s}^{-1}$, vary in single-dish linewidth from 6 to $95 \mathrm{~km} \mathrm{~s}^{-1}$, and in line flux from 25 to $300 \mathrm{Jy} \mathrm{km} \mathrm{s}{ }^{-1}$. One twelve-hour integration was obtained for each field in the standard WSRT array configuration, having a shortest baseline of 36 meters. The angular resolution retained for data presentation was about one arcmin, depending on the flux of the emission. The effective velocity resolution was 1.2 times the channel spacing of $2.06 \mathrm{~km} \mathrm{~s}^{-1}$. Examples of the WSRT imaging discussed below reveal a characteristic core/halo arrangement of $\mathrm{H}$ I fluxes, narrow linewidths, and, in several cases, a signature of rotation.

\section{Indications of a Core/Halo Morphology}

Moment images of the integrated $\mathrm{H}$ I emission, together with several representative spectra, are shown in Figures 2, 3, 4, and 6 for four of the CHVCs observed with the WSRT. In each case the emission is dominated by one or more bright knots, embedded in less intense, more diffuse gas. The linewidths in the cores are narrower than in the halos.

CHVC 069+04-223, represented in Fig. 2, illustrates the characteristic morphology. The $N_{\mathrm{HI}}$ distribution is dominated by a bright elliptical concentration (clump A) of some 15 arcmin extent; several smaller clumps to the South are 

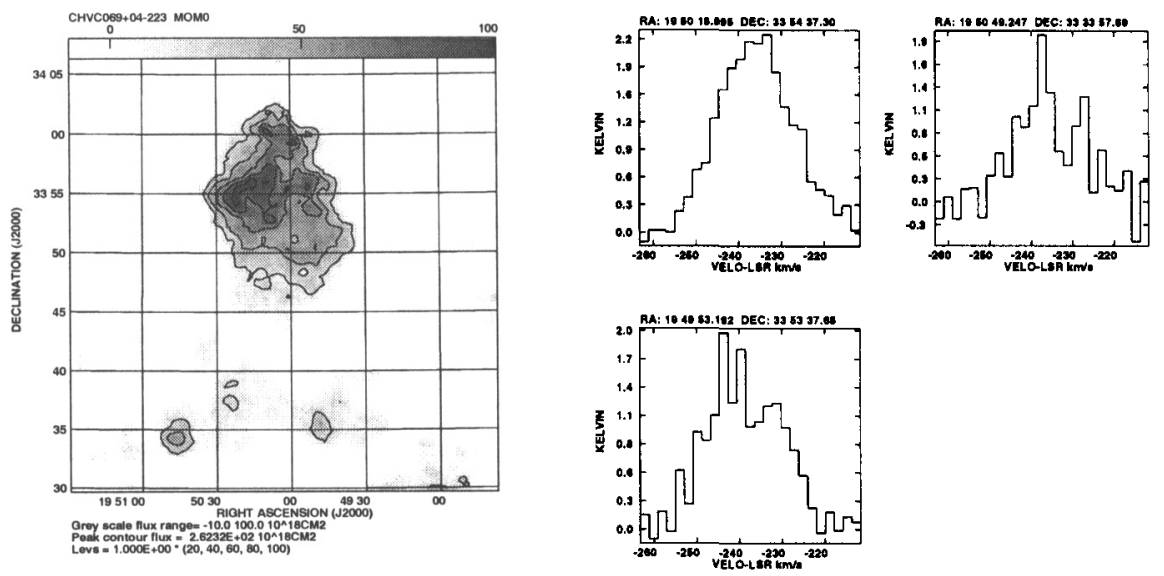

Figure 2. WSRT data for CHVC 069+04-223 at 1 arcmin and $2 \mathrm{~km} \mathrm{~s}^{-1}$ resolution. Left: apparent $N_{\mathrm{HI}}$ (assuming negligible opacity), with contours at $20,40,60,80$, and $100 \times 10^{18} \mathrm{~cm}^{-2}$ and a linear grey-scale extending from -10 to $100 \times 10^{18} \mathrm{~cm}^{-2}$. Right: brightness temperature spectra at the indicated positions.

connected by tenuous emission. (Regarding the tenuous emission, we note that while structures extending over as much as 10 arcmin in a single spectral channel were adequately recovered in the WSRT images, there were also indications in the Leiden/Dwingeloo single-dish data of more diffuse features which could not be adequately imaged by the interferometer. A straightforward attempt was made to correct the images for the weak response of the interferometer to diffuse emission features, making use of the total flux measured with the Dwingeloo telescope. The integrated $\mathrm{H}_{\mathrm{I}}$ flux detected in the reconstructed images after primary beam correction varied from less than $1 \%$ to as much as $55 \%$ of that detected in the Leiden/Dwingeloo Survey.) The velocity dispersion of the emission from the bright core is less than that from the diffuse background. The velocity field of CHVC $069+04-223$ shows a systematic velocity gradient, oriented along the major axis of clump A from about -230 to $-240 \mathrm{~km} \mathrm{~s}^{-1}$ and extending over some 10 arcmin.

The basic data for CHVC 115+13-275, represented in Fig. 3, similarly shows a core/halo morphology. This source had been completely unresolved in the single-dish data, and had shown the broadest linewidth, namely $95 \mathrm{~km} \mathrm{~s}^{-1}$, of all the CHVCs catalogued by Braun and Burton (1999). The WSRT imaging shows that this FWHM value was contributed by several separate knots of emission, each with an intrinsically much narrower velocity FWHM, amounting to about $10 \mathrm{~km} \mathrm{~s}^{-1}$. The collection of substructures, each between 1 and 10 arcmin in size, is distributed over a region of about 30 arcmin extent. Each of the cores has a distinct centroid velocity so that the collection spans the velocity interval from -300 to $-220 \mathrm{~km} \mathrm{~s}^{-1}$. Detailed examination of the WSRT data from the individual cores reveals that several of them have significant velocity gradients, oriented preferentially along their long axes. 

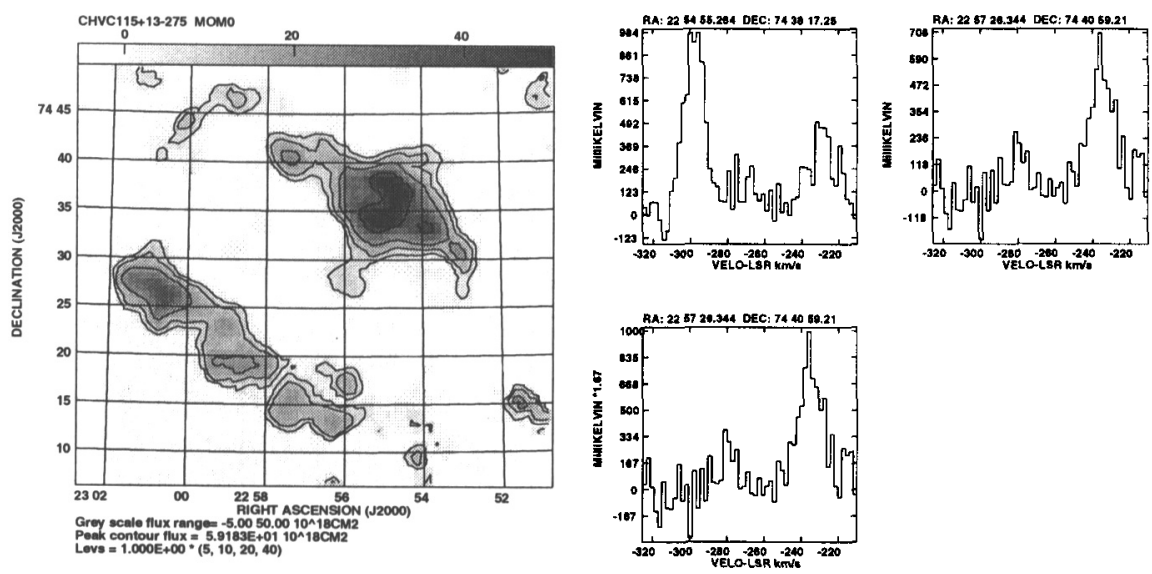

Figure 3. WSRT data for CHVC 115+13-275 at 2 arcmin and $2 \mathrm{~km} \mathrm{~s}^{-1}$ resolution. Left: apparent $N_{\mathrm{HI}}$, with contours at 5, 10 , 20 , and $40 \times 10^{18} \mathrm{~cm}^{-2}$ and a linear grey-scale extending from -5 to $50 \times 10^{18} \mathrm{~cm}^{-2}$. Right: brightness temperature spectra at the indicated positions. The velocity spread of the individually narrow cores affords a measure of distance based on dynamical crossing times.

Figure 4 shows the basic WSRT data for CHVC 204+30+075. Several relatively bright clumps are scattered over an extent of some 35 arcmin. The spectra toward the more compact local maxima have column densities of a few times $10^{20} \mathrm{~cm}^{-2}$, and FWHM widths of less than $15 \mathrm{~km} \mathrm{~s}^{-1}$. A substantial diffuse component was reconstructed for this field, reaching $N_{\mathrm{HI}}$ values of a few times $10^{19}$ $\mathrm{cm}^{-2}$ over some 30 arcmin. The large elliptical feature in the South-central part of the CHVC $204+30+075$ field (clump A) shows a well-defined velocity gradient running from about 55 to $80 \mathrm{~km} \mathrm{~s}^{-1}$ over some 12 arcmin. Similarly, the elliptical feature (clump B) in the North-East region has a velocity gradient running from 45 to $75 \mathrm{~km} \mathrm{~s}^{-1}$ over 20 arcmin.

\section{Indications of Rotational Support}

The velocity gradients mentioned above as shown by some of the CHVC cores constitute a noteworthy morphological characteristic of these objects. The gradients are oriented along the major axis of roughly elliptical $N_{\mathrm{HI}}$ distributions, and vary in amplitude from 0.5 to $2 \mathrm{~km} \mathrm{~s}^{-1} \operatorname{arcmin}^{-1}$. Examples of the signature of rotation which are particularly well resolved are clump A of CHVC 069+04-223 and clumps $\mathrm{A}$ and $\mathrm{B}$ of CHVC $204+30+075$. These are reminiscent in form and amplitude of the "spider" diagrams portraying the $\mathrm{H}$ I kinematics of some dwarf galaxies. Consequently we carried out standard tilted-ring fits to assess the extent to which the CHVC kinematics could be modeled by rotation in a flattened disk system. 

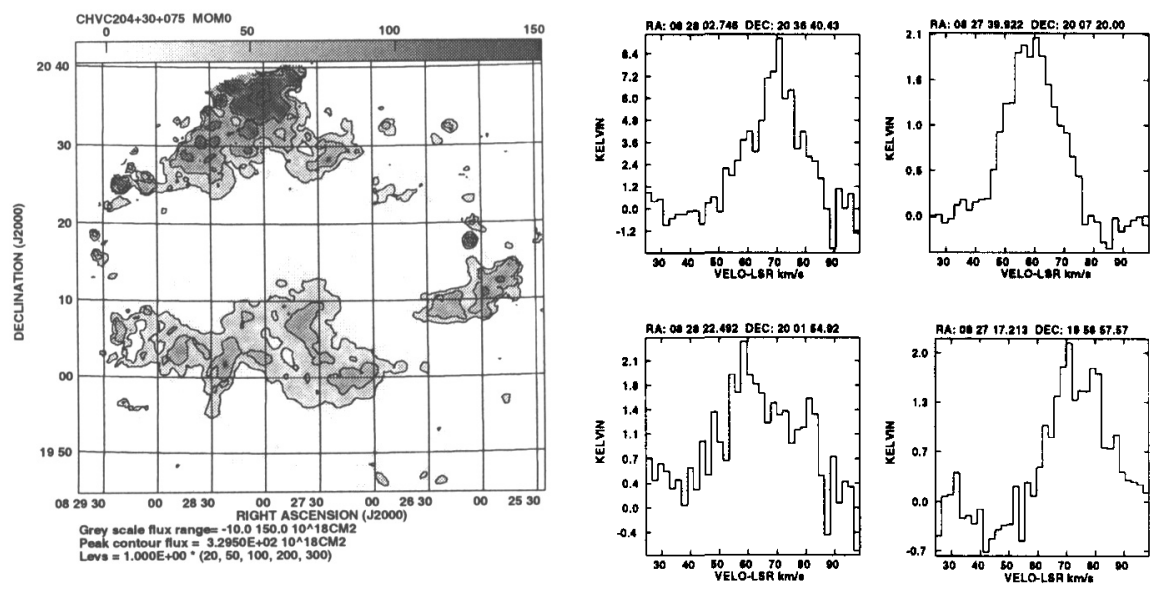

Figure 4. WSRT data for CHVC $204+30+075$ at 1 arcmin and $2 \mathrm{~km} \mathrm{~s}^{-1}$ resolution. Left: apparent $N_{\mathrm{HI}}$, with contours at 20,50 , 100,200 , and $300 \times 10^{18} \mathrm{~cm}^{-2}$ and a linear grey scale extending from -10 to $150 \times 10^{18} \mathrm{~cm}^{-2}$. Right: brightness temperature spectra at the indicated positions.

Figure 5 shows that robust solutions for circular rotation pertain for the three cases. The fits indicate rotation velocity rising slowly and continuously with radius, to some $15 \mathrm{~km} \mathrm{~s}^{-1}$ in the case of CHVC $069+04-223 \mathrm{~A}$, and flattening out to values of 15 and $20 \mathrm{~km} \mathrm{~s}^{-1}$ for CHVC $204+30+075 \mathrm{~A}$ and $\mathrm{B}$, respectively. An estimate of the total mass supporting this rotation follows from $M_{\mathrm{dyn}}=R v^{2} / \mathrm{G}=2.3 \times 10^{5} R_{\mathrm{kpc}} v_{\mathrm{km} / \mathrm{s}}^{2}$. At an assumed distance of $0.7 \mathrm{Mpc}$, these cores have $M_{\mathrm{dyn}}=10^{7.1}, 10^{6.5}$, and $10^{6.9} \mathrm{M}_{\odot}$, respectively. The mass of the gas, assuming a $40 \%$ contribution by helium, follows from $M_{\mathrm{gas}}=1.4 M_{\mathrm{HI}}=$ $3.2 \times 10^{5} S D_{\mathrm{Mpc}}^{2}$, where $S$ is the integrated H I flux in units of $\mathrm{Jy} \mathrm{km} \mathrm{s}^{-1}$. For these three cores, $M_{\text {gas }}=10^{7.1}, 10^{6.5}$, and $10^{6.9}$, values which, compared to the dynamical masses, correspond to dark-to-visible mass ratios of 10,36 , and 29 .

\section{Considerations of CHVC Stability}

The very high total linewidth of CHVC 115+13-275 was found to arise from the range of line-of-sight velocities contributed by the distinct individual clumps which make up this source. It is unlikely that this is a chance superposition of unrelated components, in view of the spatial and kinematic isolation of this CHVC. Braun and Burton (2000) consider the stability of these collection of clumps under several circumstances. If the collection of clumps were located at a distance of $5 \mathrm{kpc}$, the collection would have a diameter of $44 \mathrm{pc}$ and would double in size on the implausibly short dynamical timescale of only $5 \times 10^{5}$ years. On the other hand, if this source were located at a distance of $0.7 \mathrm{Mpc}$ and were self-gravitating, then the angular radius of 15 arcmin and the velocity half- 

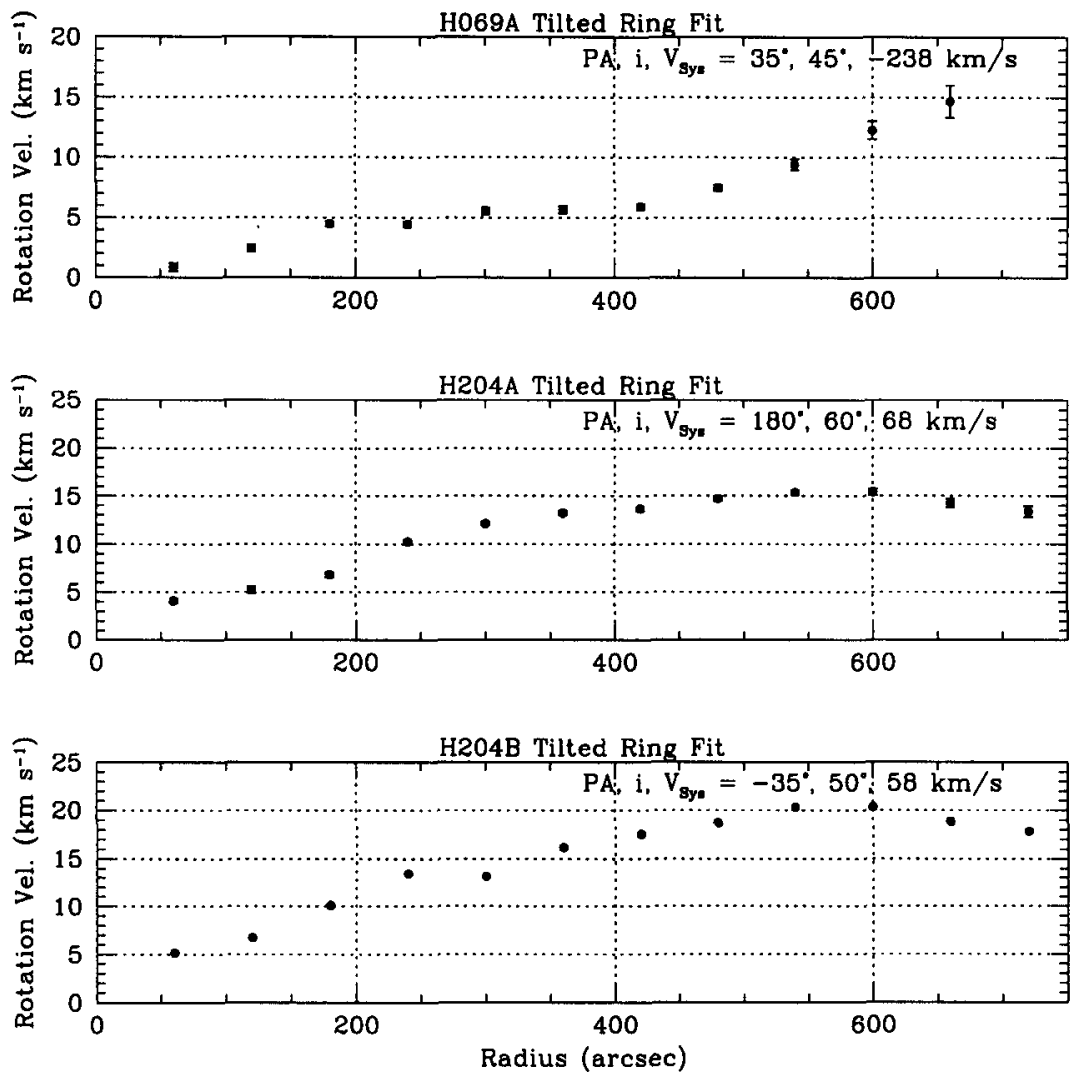

Figure 5. Derived rotation velocities in three elliptical cores, CHVC $069+04-223 \mathrm{~A}$ and CHVC $204+30+075 \mathrm{~A}$ and B. The bestfitting position angle, inclination, and systemic velocity are indicated in each panel. These were subsequently held fixed in deriving the rotation velocity as function of radius. These CHVC cores are evidently rotationally supported. The dynamical masses derived for the cores assuming a distance of $0.7 \mathrm{Mpc}$ are $10^{8.1}, 10^{8.1}$, and $10^{8.3} \mathrm{M}_{\odot}$; the dark-to-visible mass ratios are 10,36 , and 29 , respectively. 
width of $35 \mathrm{~km} \mathrm{~s}^{-1}$ could be used to calculate a dynamical mass of $10^{8.93} \mathrm{M}_{\odot}$. The corresponding mass of gas at this distance, assuming $40 \%$ to be helium, is $10^{7.22} \mathrm{M}_{\odot}$, and the dark-to-visible mass ratio is 51 and scales as $1 / \mathrm{D}$. Consistent distance and dark-to-visible mass ratios are found for other CHVC objects.

\section{Indications of Cold, Opaque Cores}

The object CHVC 125+41-207 is particularly interesting. Of the 65 CHVCs catalogued by Braun and Burton (1999), this one showed the narrowest FWHM, amounting to $5.9 \mathrm{~km} \mathrm{~s}^{-1}$ in the single-dish data. The WSRT image shown in Fig. 6 could be displayed at higher angular resolution because of the intense brightness of this source. Like the other CHVCs imaged, this one also shows a complex morphology with several compact cores. The spectrum toward the brightest of these cores is remarkable in having a linewidth which is completely unresolved with the effective resolution of $2.47 \mathrm{~km} \mathrm{~s}^{-1}$. The velocity channels adjacent to the line peak have intensities down to about $20 \%$ of the maximum value. Such a width is one of the narrowest ever measured in $\mathrm{H} \mathrm{I}$ emission.

The narrow linewidth provides an opportunity (rare in $\mathrm{HI}$ work, where linewidths are commonly dominated by line blending and mass motions) to derive a kinetic temperature. An upper limit to the intrinsic FWHM of $2 \mathrm{~km} \mathrm{~s}^{-1}$ corresponds to an upper limit to the kinetic temperature of $85 \mathrm{~K}$. This limit assumes additional importance because the observed brightness temperature of this core is $75 \mathrm{~K}$. Thus the temperature is tightly constrained, and a lower limit to the opacity follows from $T_{\mathrm{B}}=T_{\mathrm{s}}\left(1-e^{-1}\right)$, if $T_{\mathrm{s}}=T_{\mathrm{k}}$, yielding $\tau \geq 2$. In addition to constraining the temperature, this observation also constrains any broadening which might be due to turbulence to be less than $1 \mathrm{~km} \mathrm{~s}^{-1}$.

The narrow linewidth observed for CHVC 125+41-207 provides an opportunity to estimate its distance. Wolfire et al. (1995a, 1995b) show that a cold, stable phase of $\mathrm{H}$ I is expected if a sufficient column of shielding gas is present and if the thermal pressure is sufficiently high. Calculations of equilibrium $\mathrm{H}$ I conditions with the Local Group environment have kindly been made available to us by Wolfire, Sternberg, Hollenbach, and McKee, for two bracketing values of the shielding column density, namely 1 and $10 \times 10^{20} \mathrm{~cm}^{-2}$. Figure 7 shows that the corresponding equilibrium volume densities for the observed value of $T_{\mathrm{k}}=85 \mathrm{~K}$ are 3.5 and $0.65 \mathrm{~cm}^{-3}$, respectively. The distance to CHVC $125+41-207$ follows from the volume densities thus indicated, and from the measured column depths and the angular size of the source, according to $D=N_{\mathrm{H}} /\left(n_{\mathrm{H}} \theta\right)$. Consideration in this way of several compact cores in this source allow a distance determination in the range 0.5 to $1 \mathrm{Mpc}$.

\section{Summary: Parameters of Several of the Individual Objects in the CHVC Ensemble}

The compact high-velocity clouds which we have imaged using the Westerbork array share several morphological characteristics. In each case we detected a number of compact cores which range in size from a few arcmin to about 15 arcmin, with peak column densities in the range $10^{19}$ to $10^{20} \mathrm{~cm}^{-2}$, and FWHM linewidths of about $2 \mathrm{~km} \mathrm{~s}^{-1}$. Each core is characterized by its own line-of-sight 


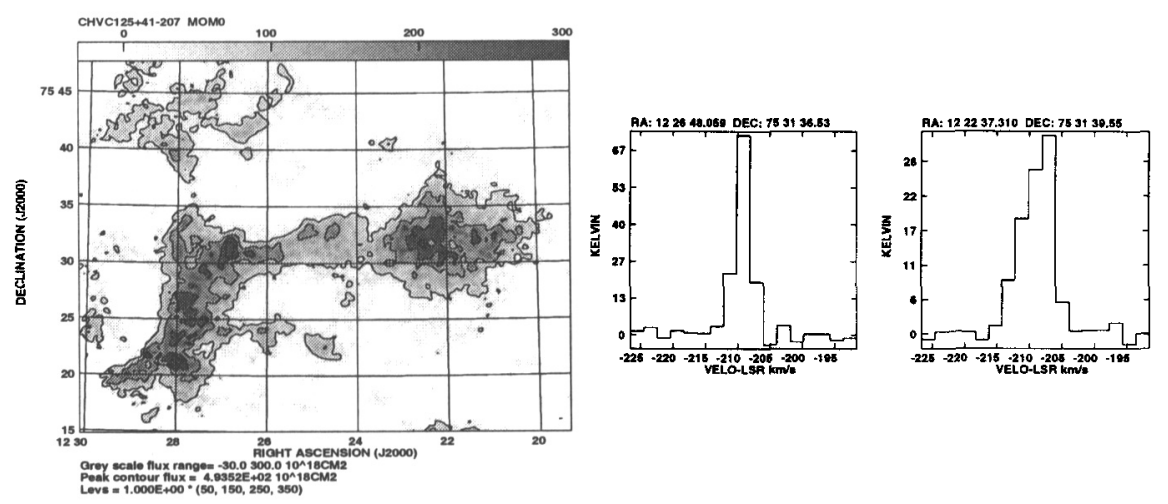

Figure 6. WSRT data for CHVC 125+41-207 at 28 arcsec and $2 \mathrm{~km} \mathrm{~s}^{-1}$ resolution. Left: apparent $N_{\mathrm{HI}}$, with contours at 50,150, 250 , and $350 \times 10^{18} \mathrm{~cm}^{-2}$ and a linear grey-scale extending from -30 to $300 \times 10^{18} \mathrm{~cm}^{-2}$. Right: brightness temperature spectra at the indicated positions. The spectrum shown on the left in this panel is one of the narrowest $\mathrm{H}$ I emission lines ever observed. The extremely narrow linewidths robustly constrain the kinetic temperatures as well as the turbulence.

velocity; it is the range in these core velocities, not the much narrower intrinsic linewidths, which determined the range of total widths in the single-dish catalog of CHVCs. The modest linewidths seen in all of the cores indicates that the $\mathrm{H} I$ in these structures is in the form of the Cool Neutral Medium (CNM) with typical equilibrium temperatures in the range 50 to $200 \mathrm{~K}$.

In all of the cases we have imaged, as well as in the two cases imaged earlier by Wakker and Schwarz (1991), the CHVC cores are embedded in an extended halo of $\mathrm{HI}$ emission. The halos have typical HI extents of about $1^{\circ}$, column densities within the inner 30 arcmin between $2 \times 10^{18}$ and $2 \times 10^{19} \mathrm{~cm}^{-2}$, and velocity dispersions consistent with the $8000 \mathrm{~K}$ equilibrium temperature of the Warm Neutral Medium (WNM). The cores account for some $40 \%$ of the $\mathrm{H}$ I flux, while covering some $15 \%$ of the area. Thus the data at hand suggest that a twophase structure characterizes the CHVC morphology, with cold, opaque cores of CNM shielded by a halo of the WNM.

The compact clouds provide several indications of the crucial distance parameter. The velocity dispersion of the entire ensemble is minimized in a reference frame consistent with the Local Group Standard of Rest. Three additional measures regarding distance are given by the imaging of individual CHVCs. (i) Arguments based on the dynamical stability of CHVC 115+13-275 support a distance of about $0.7 \mathrm{Mpc}$. (ii) The exceptionally narrow linewidths observed in the principal cores of CHVC 125+41-207 lead to a measure of the volume density and that, with the measured angular size and column depth, yields a distance in the range 0.5 to $1 \mathrm{Mpc}$. (iii) The kinematic gradients detected along the long axis of some of the CHVC cores resemble in form and amplitude the velocity fields of dwarf galaxies. The solutions for dynamical mass based on this 


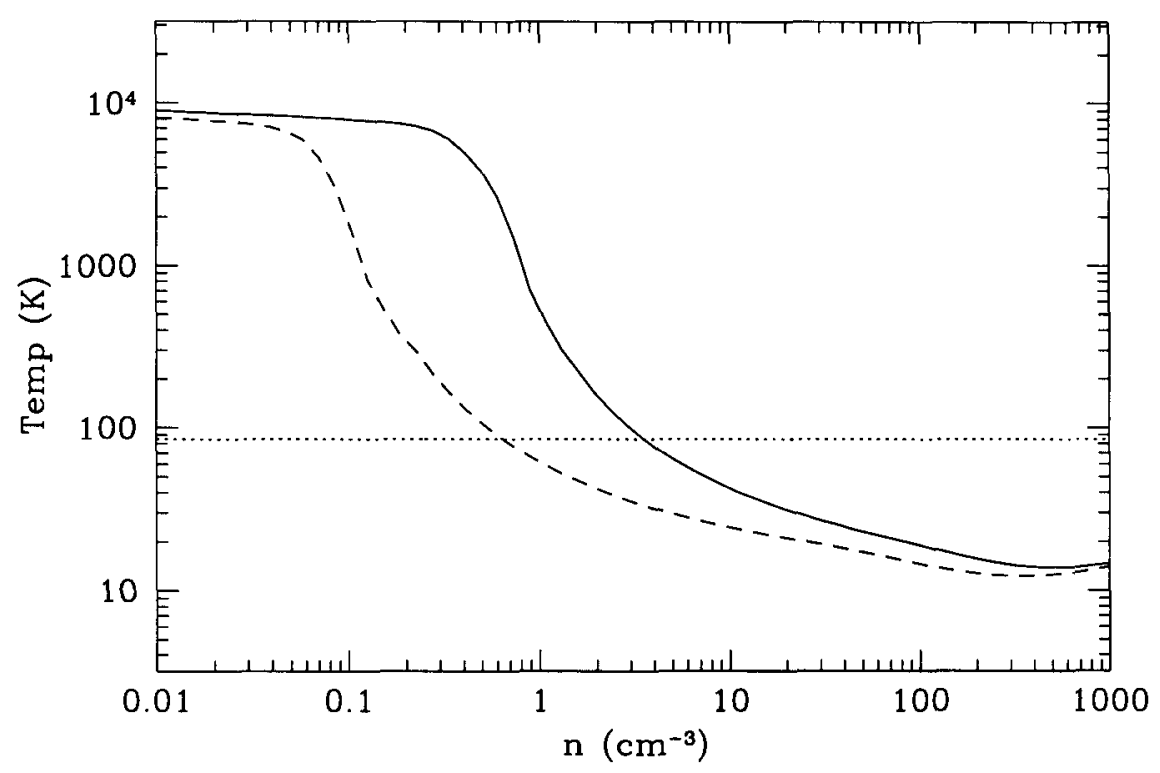

Figure 7. Equilibrium temperature curves for $\mathrm{HI}$ in an intergalactic radiation field at a metallicity of 0.1 solar and a dust-to-gas mass ratio of 0.1 times the value representative of the solar neighborhood, and for two values of the neutral shielding column density, namely $10^{19} \mathrm{~cm}^{-2}$, indicated by the solid line, and $\times 10^{20} \mathrm{~cm}^{-2}$, indicated by the dashed line. The $85 \mathrm{~K}$ kinetic temperature of the opaque cores in CHVC $125+41-207$ is indicated by the dotted line.

comparison indicate a distance of about $0.7 \mathrm{Mpc}$. The ratio of dark-to-visible mass of the objects is in the range 10 to 50 .

In agreement with the conclusions reached for the ensemble, the distances and physical properties of the individual CHVCs are suggestive of a population which has as yet had little interaction with the more massive Local Group members. At distances of about 0.5 to $1 \mathrm{Mpc}$ these objects would have sizes of about $15 \mathrm{kpc}$, gas masses of about $10^{7} \mathrm{M}_{\odot}$, and total masses of a few times $10^{8} \mathrm{M}_{\odot}$. We tentatively estimate the total CHVC ensemble to harbor some 200 objects; in that case, the total gas mass involved would be a few times $10^{9} \mathrm{M}_{\odot}$. In view of the net in-fall motion observed for the ensemble, a source of dark matter and low-metallicity gas is indicated for the continuing growth and evolution of the major Local Group galaxies. The CHVCs may be the missing Local Group satellites predicted by hierarchical growth scenarios (e.g. Klypin et al. 1999).

These CHVC parameter values correspond to those of (sub-)dwarf galaxies. Indeed, it would be difficult to distinguish these CHVC properties from those derived from the $\mathrm{H}$ I signature of a typical dwarf galaxy. If, on the other hand, the objects were produced relatively locally by an energetic mechanism responsible for a galactic fountain, then their $\mathrm{H}$ I properties would be expected to show large linewidths, motions not ordered by rotation, and a structural form other than a core/halo one. The distinction between CHVCs and dwarf galaxies with very 
weak star formation remains to be made and is an important challenge. The CHVCs may represent still-pristine examples of collapsed objects, with only a small amount of internal star formation and enrichment. As such, they should provide insight into the process of galaxy and structure formation.

The WSRT imaging is discussed in more detail by Braun and Burton (2000).

Acknowledgments. We are grateful to M.G. Wolfire, A. Sternberg, D. Hollenbach, and C.F. McKee for providing the equilibrium temperature curves shown in Fig. 7. The Westerbork Synthesis Radio Telescope is operated by the Netherlands Foundation for Research in Astronomy, under contract with the Netherlands Organization for Scientific Research.

\section{References}

Bajaja E., Morras R., Pöppel W.G.L. 1987, P. A. Inst. Czech. Ac. Sci. 69, 237

Blitz L., Spergel D. N., Teuben P. J., Hartmann D., Burton W.B. 1999, ApJ, 514,818

Braun R., Burton W. B. 1999, A\&A,341, 437

Braun R., Burton W. B. 2000, A\&A, submitted

Bregman J.N. 1980, ApJ, 236, 577

Burton W. B. 1997, in The Physics of the Galactic Halo, eds. Lesch H., Dettmann R.-J., Mebold U., Schlickeiser R., Berlin: Akademie Verlag, 15

Eichler D. 1976, ApJ, 208, 694

Einasto J., Haud U., Jôeveer M., Kaasik A. 1976, MNRAS, 177, 357

Giovanelli R. 1981, AJ, 86, 1468

Hartmann D., Burton W. B. 1997, "Atlas of Galactic Neutral Hydrogen", Cambridge: Cambridge University Press

Karachentsev I. D., Makarov D. A. 1996, AJ, 111, 794

Klypin A., Kravtsov A. V., Valenzuela O., Prada F. 1999, ApJ, 522, 82

Moore B., Ghigna S., Governato G., Lake G., Quinn T., Stadel J., Tozzi P. 1999, ApJ, 524, 19

Oort J.H. 1966, Bull. Astr. Inst. Netherlands, 18, 421

Oort J.H. 1970, A\&A, 7, 381

Oort J.H. 1981, A\&A, 94, 359

Shapiro P. R., Field G. B. 1976, ApJ, 205, 762

Verschuur G. L. 1975, ARA\&A, 13, 257

Wakker B. P., Schwarz U. 1991, A\&A, 250, 484

Wakker B. P., van Woerden H. 1991, A\&A, 250, 509

Wakker B. P., van Woerden H. 1997, ARA\&A, 35, 217

Wolfire M. G., Hollenbach D., McKee C. F., Tielens A. G. G. M., Bakes E. L. O. 1995a, ApJ, 443, 152

Wolfire M. G., McKee C. F., Hollenbach D., Tielens A. G. G. M. 1995b, ApJ, 453,673 